\title{
Crossing the quality chasm in resource-limited settings
}

\author{
Duncan Smith-Rohrberg Maru 1,2,3, , Jason Andrews $^{1,7}$, Dan Schwarz ${ }^{1,2,3}$, Ryan Schwarz ${ }^{1,2,3}$, Bibhav Acharya ${ }^{1,4}$, \\ Astha Ramaiya', Gregory Karelas', Ruma Rajbhandari ${ }^{1,2}$, Kedar Mate ${ }^{2,5,6}$ and Sona Shilpakar ${ }^{1}$
}

\begin{abstract}
Over the last decade, extensive scientific and policy innovations have begun to reduce the "quality chasm" - the gulf between best practices and actual implementation that exists in resource-rich medical settings. While limited data exist, this chasm is likely to be equally acute and deadly in resource-limited areas. While health systems have begun to be scaled up in impoverished areas, scale-up is just the foundation necessary to deliver effective healthcare to the poor. This perspective piece describes a vision for a global quality improvement movement in resource-limited areas. The following action items are a first step toward achieving this vision: 1) revise global health investment mechanisms to value quality; 2) enhance human resources for improving health systems quality; 3) scale up data capacity; 4) deepen community accountability and engagement initiatives; 5) implement evidence-based quality improvement programs; 6) develop an implementation science research agenda.
\end{abstract}

Keywords: Resource-limited, Health system, Global health, Quality improvement

\section{The quality chasm in resource-limited settings}

A young school-age boy with severe respiratory distress presented to the remote hospital. Previously, the boy had been seen by untrained private clinicians in the community three times over four days. At presentation to the hospital, the child was evaluated by a mid-level practitioner who provided an initial course of antibiotics. Despite the child's ill appearance, supportive treatment including intravenous fluids and supplemental oxygen were not provided until discussion with the Medical Director three hours later. Later that evening, the electric nebulizer and oxygen concentrator became unusable after the hospital lost power owing to a blackout of the public electric grid and malfunctioning of the hospital's backup generator. The regulator for the backup oxygen canister could not be found. At this juncture, without the ability to provide oxygen, the medical team recommended transfer. The family did not agree to transfer due to the high costs of other regional health facilities (our facility provides free services). That evening, after not being examined for over two hours by on-call staff,

\footnotetext{
* Correspondence: duncansr@post.harvard.edu

${ }^{1}$ Nyaya Health, Bayalpata Hospital, Ridikot VDC, Achham, Nepal

'Brigham and Women's Hospital, Department of Medicine, Boston, MA, USA Full list of author information is available at the end of the article of the article
}

the child was found unresponsive with a thready pulse. Cardiopulmonary resuscitation was not initiated for over ten minutes as the midwife managing the ward did not know the procedure and the bag valve mask was not at the bedside. Following fifteen minutes of unsuccessful resuscitation, the child was declared dead.

Health care providers in resource-limited settings must do better [1]. Globally, resource-limited settings have received increasing funds over the last decade for the scaling up of health programs. These resources have generally been focused on the quantity of services provided. The quality of many of the resultant services, however, has often times been low or poorly understood. Since the 1980s, quality improvement (QI) research and implementation have taken steps towards reducing the "quality chasm" $[2,3]$ that exists in developed-country settings [4-7]. If increased funding mechanisms for disease-focused global health initiatives was the major theme of the last decade in global health policy [8], then the next decade must emphasize the quality of health systems, and indeed several global initiatives are now underway $[9,10]$. Across all six domains of healthcare quality [2] - safety, effectiveness, efficiency, patientcenteredness, timeliness, and equity-bridging the global quality chasm requires reconceptualizing the delivery of
C Biomed Central

(c) 2012 Maru et al.; licensee BioMed Central Ltd. This is an Open Access article distributed under the terms of the Creative Commons Attribution License (http://creativecommons.org/licenses/by/2.0), which permits unrestricted use, distribution, and reproduction in any medium, provided the original work is properly cited. 
health services to the global poor. The experiences of clinicians delivering health care in rural regions have made clear the urgent need for new strategies. These strategies must address logistical, supply chain, engineering, human resources and traditional clinical challenges, while remaining cognizant of and adaptable to the unique medical and socio-cultural needs of each setting. While there are many local and global initiatives currently ongoing in this area, there is a need for a concise vision from the view of grassroots providers. Towards this end, this perspective paper describes one vision for a global quality improvement movement (Additional file 1: Figure S1.

If such a movement were to take hold, how might the young school-age boy have fared differently? Let us reenvision the case with a different outcome, one that we propose a global QI movement could achieve. One day after his parents reported a cough and fever, the boy was visited by a community health worker and referred to a health post where he received an initial course of antibiotics. On the second day, when follow-up revealed a higher fever and respiratory distress, the child was referred to the hospital. After initial consultation, a midlevel provider admitted the patient and spoke directly with the Medical Director to ensure proper management. Oxygen was administered via a generator-powered nebulizer, and on the third hospital day, after significant decline, the child was transferred via an oxygenequipped ambulance to a regional hospital, where intensive care unit staff were expecting the referral. After eight days on the inpatient unit at the regional hospital, the child followed up as an outpatient and was visited at home regularly by a community health worker. The following week, the family attended a community health worker class on respiratory illness and prevention in the community.

\section{A global quality improvement movement}

The following broad categories will be the pillars of an effective global QI movement: 1) revise global health investment mechanisms to value quality; 2) enhance human resources for improving health systems quality; 3 ) scale up data capacity; 4) deepen community accountability and engagement initiatives; 5) implement evidencebased QI programs; 6) develop an implementation science research agenda.

\section{Revise global health investment mechanism to value quality}

The dominant approach to global health financing remains tied to volume-based targets, such as the number of patients provided family planning services or patients initiated on antiretroviral treatment [11]. Emphasizing quality metrics in the planning and financing of health services could alter the incentives of health systems actors to provide better quality, not just quantity, healthcare. Several individual projects and financing schemes have developed quality metrics, but a more concerted, cultural shift is required [11,12]. Investments in quality health systems will take longer to bear fruit, so donors will have to adapt their mindsets and establish interim benchmarks for longer-term objectives. In the case of this hospital, we would have access to additional training programs, and would be monitored by district authorities and local community development committees to improve quality, if central funding mechanisms were revised with an eye towards quality.

\section{Enhance human resources for improving health systems quality}

Global healthcare faces crisis-level personnel shortages, which make developing and sustaining QI initiatives extremely difficult [13]. In addition to recruitment and retention initiatives, the development of a global QI movement will require further focus on QI-related medical education. Since many developing nation medical education centers are currently unequipped to effectively teach QI $[14,15]$, developing QI-related curricula, continuing medical education programs and regular competence evaluations and self-assessments for all levels of healthcare providers [16-18] will be critical. Moreover, a particular focus on rural facilities where personnel have little, if any, interaction with academia or continuing educational efforts would be important. QI initiatives also lead to improved system performance and efficiency improving health workforce productivity and boosting morale.

In the case of the young school-age boy, more robust QI-related education during staff training, and regular staff competence review in our hospital could have identified and addressed many of the issues that resulted in this boy's death. For example, performance evaluations of our nurse midwives would have documented insufficient knowledge of resuscitation protocols, enabling targeted education to improve resuscitation quality.

\section{Scale up data capacity}

Health systems learn much like people do by receiving constructive and context-specific feedback on their performance. As such, a culture of real-time data collection and evaluation is crucial to the QI endeavor [19]. Data systems are difficult to establish in resource-limited settings, due to low computer literacy, unreliable electricity, and competing financial needs that supercede information technology investments. Basic reporting schemes are often incomplete or inaccurate, and are not analyzed in a provider-friendly format. Moreover, most reporting systems rely on inefficient paper systems and lack the 
infrastructure to upgrade to electronic versions [20]. Importantly, most clinics and health care providers who generate data for public health reporting rarely receive feedback on their performance. For information to drive clinical practice, data systems need to be timely, accurate, accessible, and relevant. They need not and should not be particularly complex. To avoid duplication with existing national data systems, the collection and uses of data should be reviewed and QI metrics should be incorporated within national data systems. These data systems should be developed to feedback to providers with information on their performance, comparison to other facilities, and areas for improvement.

In the case of the young school-age boy, data on pneumonia treatment in our facility will help inform the clinical practice and process improvements we make. To evaluate our pneumonia management we will need to know, for example, rates of febrile children for whom a respiratory rate was documented, action taken based upon established protocols (e.g., Integrated Management of Childhood Illness [21]), and rates of appropriate antibiotic prescription.

\section{Deepen community accountability and engagement initiatives}

Accountability practices have been shown to enhance QI in numerous settings, yet are significantly lacking globally. In many resource-limited settings, low education rates paralleled by unequal power dynamics between health providers and patients make accountability challenging. Strategies to address these issues include holding public meetings about available services [22], making performance data publicly available [23], communityaccountability checklists, and publishing health institution report cards [24].

All of these serve to engage patients and community members in taking ownership over quality of healthcare for their citizens. Community groups will need to be supported with both financial and technical assistance to ensure that they have the resources to provide excellent monitoring of the quality of the healthcare they receive. The overall goal is to inform the communities each health facility serves, augmenting efforts for constant service improvement and informed community decision making. In the case of the young school-age boy, knowing the performance metrics of untrained care providers might have influenced his parents to bring him directly to the hospital, before his condition had reached an emergency level.

\section{Implement evidenced-based quality improvement programs}

There is an acute need to close the "implementation gap" in global healthcare delivery $[25,26]$-that disconnect between what is known and what is actually available in resource-limited settings. Some approaches unique to resource-limited environments may be needed, but while we await the research and development of such strategies, successful QI paradigms utilized in resource-rich contexts could be adapted and implemented. The following nonexhaustive list outlines a few simple ways to implement QI practices within diverse and resource-limited settings.

The Plan-Do-Study-Act (PDSA) model is a component of the well-tested Model for Improvement. The PDSA could help meet the lack of critical reflection and operations improvement within global health [27]. Within PDSA, straightforward cause-and effect process mapping through Ishikawa or other diagrams could engage providers at all educational levels, and enable critical staff buy-in within resource-limited healthcare institutions. This model can be applied in the clinical, outreach, managerial, logistical, supply chains, and engineering realms of healthcare management. Other QI tools like the Breakthrough Series College can assist program designers and health systems planners to more rapidly spread best practice throughout entire districts and even countries [28,29].

Checklists designed to ensure standardized, high quality care and team communication improve the effectiveness of clinical care in resource-limited settings [4,30]. Checklists are a low-tech solution that can bolster managerial oversight and better utilize available human resources, improving overall effectiveness. Checklists are an important tool for standardization but their effective implementation will have to undergo rigorous sitespecific testing using QI principles.

Morbidity and mortality (M\&M) case conferences can ensure both ongoing clinical education and regular, inclusive, team-based process evaluation [31]. This has been our experience in rural Nepal, where health personnel typically have few opportunities for continuing medical education or team-based reflection. M\&Ms in other locations have also been transformed into QI meetings where district/subdistrict process and outcome data are shared with a community of clinics and hospitals to assist with data feedback.

Multidisciplinary care teams for patients with chronic medical conditions have also improved quality in diverse settings $[32,33]$. Community health workers should form the foundations of these teams [34]. Much of the primary care system infrastructure in developing countries was designed to manage acute ailments and functions as a network of urgent instead of primary care centers. Multidisciplinary teams can help change this dynamic.

If implemented broadly and adapted appropriately, such strategies would ultimately offer a rubric of standard high quality healthcare practices to diverse resourcelimited environments. Many of the errors that occurred 
in our patient's care could have been avoided by applying basic, low-tech practices such as these.

\section{Develop an implementation science research agenda}

An implementation science research agenda is crucial to advancing the field and practice of quality improvement. Research into quality in global health has historically focused on the efficacy of interventions demonstrated through large-scale randomized controlled trials and subsequent guideline development. The new implementation science research agenda must focus on understanding implementation in context which requires new context-specific evaluation tools like time-series analysis, statistical process control or adaptive clinical trials. Also, the areas of investigation should not center on new therapeutic strategies tested in controlled environments but the application of known strategies in diverse, uncontrolled environments.

Furthermore, research is needed not just on clinicallyfocused problems but also on logistical and managerial ones $[35,36]$. Waste, water quality, energy, infectious disease control, supply chains, human resource management systems all require study, reflection, and scrutiny. This research should be coordinated, tied to real practice, and focused on both outcomes and processes. A central research methodology will include in-depth qualitative and descriptive quantitative analyses of the implementation of and longitudinal follow-up of individual QI projects. This should be supplemented by more costly randomized controlled trials comparing QI interventions and non-randomized multi-site outcomesoriented longitudinal studies. Through such research, we can help rectify the logistical, communication, and managerial failures that led to the death of the young school-age boy.

\section{A global quality improvement movement}

With the aim of achieving improved outcomes for patients globally, we have underscored the importance of a global healthcare QI movement and outlined initial steps toward it. The far-reaching goals of global health do not stop with health systems scale-up. Rather, scaleup is the foundation of a larger movement aimed at delivering quality healthcare services for all. To achieve this vision, we will need new resources and a concerted, quality-oriented agenda. Many of these interventions will be relatively inexpensive, though data on the cost and effectiveness of these interventions is currently unknown; as the movement develops, efforts will be needed to centralize these data to ensure cost-effective use of resources.

We can save lives and prevent disability by improving the quality of healthcare services in the resource-limited settings. The immediate actions described in this article towards achieving healthcare quality improvement are important. It may be overwhelming and impractical to achieve all quality improvement measures in a short time. However, if actions are taken, quality improvement can be achieved in the long term. Collective efforts from funding sources as well as healthcare consumers would help to achieve this common goal of healthcare quality improvement in resource-limited settings.

\section{Additional file}

Additional file 1: Figure S1. Strategies for a Global Quality

Improvement Movement.

\section{Abbreviations}

QI: Quality improvement; PDSA: Plan-Do-Study-Act; M\&M: Morbidity and mortality.

\section{Competing interests}

The authors declare that there is no financial or non-financial competing interest.

\section{Authors' contributions}

The authors are professionals who work in varying capacities with Nyaya Health, a non-profit group delivering healthcare services in rural Nepal that is innovating methods of open-access and transparency in the field of global health. Together, they have conceptualized this piece as a reflection upon their collective experiences within Nepal and individual ones in other resource-limited areas. All authors reviewed and provided inputs and edits to the final piece. DM conceived the piece, wrote the initial draft, and serves as the guarantor of the article. All authors read and approved the final manuscript.

\section{Authors' information}

Authors are affiliated with the Bayalpata Hospital which is located in far west of Nepal. The case presented in this article was the case encountered at the Bayalpata Hospital. Based upon author's experiences delivering health care in resource limited-setting, authors propose the actions that needs to be taken to achieve healthcare quality improvement in resource-limiting settings.

\section{Acknowledgements}

The global health quality improvement movement described in this article is in context of the working experience at the resource-limited region of Nepal. This study received no financial support.

\section{Author details}

${ }^{1}$ Nyaya Health, Bayalpata Hospital, Ridikot VDC, Achham, Nepal. ${ }^{2}$ Brigham and Women's Hospital, Department of Medicine, Boston, MA, USA.

${ }^{3}$ Children's Hospital of Boston, Department of Medicine, Boston, MA, USA. ${ }^{4}$ Department of Psychiatry, University of California San Francisco, School of Medicine, San Francisco, CA, USA. Institute for Healthcare Improvement, Cambridge, MA, USA. ${ }^{6}$ Department of Medicine at Weill Cornell Medical College, New York, NY, USA. ${ }^{7}$ Division of Infectious Diseases, Massachusetts General Hospital, Boston, MA, USA. ${ }^{8}$ Global Health Equity Program Brigham and Women's Hospital and Children's Hospital of Boston, Boston, USA.

Received: 28 December 2011 Accepted: 15 November 2012 Published: 30 November 2012

\section{References}

1. Kim JY, Rhatigan J, Jain SH, Weintraub R, Porter ME: From a declaration of values to the creation of value in global health: a report from Harvard University's Global Health Delivery Project. Global Public Health 2010, 5:181-188.

2. Crossing the Quality Chasm: A New Health System for the 21st Century. [http://books.nap.edu/openbook.php?record_id=10027]. 
3. Kohn LT, Corrigan J, Donaldson MS: Institute of Medicine (U S) Committee on Quality of Health Care in America. Natl Academy Pr: editor: To err is human; 2000.

4. Haynes AB, Weiser TG, Berry WR, Lipsitz SR, Breizat AH, Dellinger EP, Herbosa T, Joseph S, Kibatala PL, Lapitan MC, Merry AF, Moorthy K, Reznick RK, Taylor B, Gawande AA: A surgical safety checklist to reduce morbidity and mortality in a global population. N Engl J Med 2009, 360:491-499.

5. Ellsbury D: Crossing the quality chasm in neonatal-perinatal medicine. Clin Perinatol 2010, 37:1.

6. White $\mathrm{HL}$, Glazier $\mathrm{RH}$ : Do hospitalist physicians improve the quality of inpatient care delivery? A systematic review of process, efficiency and outcome measures. BMC Med 2011, 9:58.

7. Nyamtema $A S$, de Jong $A B$, Urassa DP, van Roosmalen J: Using audit to enhance quality of maternity care in resource limited countries: lessons learnt from rural Tanzania. BMC Pregnancy Childbirth 2011, 11:94.

8. World Health Organization Maximizing Positive Synergies Collaborative Group, Samb B, Evans T, Dybul M, Atun R, Moatti JP, Nishtar S, Wright A Celletti F, Hsu J, Kim JY, Brugha R, Russell A, Etienne C: An assessment of interactions between global health initiatives and country health systems. Lancet 2009, 373:2137-2169.

9. Travis $P$, Bennett $S$, Haines A, Pang T, Bhutta Z, Hyder AA, Pielemeier NR, Mills A, Evans T: Overcoming health-systems constraints to achieve the Millennium Development Goals. Lancet 2004, 364:900-906.

10. $\mathrm{WHO} \mid$ Health systems strengthening: $\mathrm{WHO} \mid$ Health systems strengthening [http://www.who.int/healthsystems/en/]

11. Annual Report to Congress on PEPFAR Program Results: PEPFAR:; 2009.

12. USAID Health Care Improvement Project ( $\mathrm{HCl})$ | Global: [http://www.urc-chs. com/project?ProjectID=34].

13. Anyangwe $\mathrm{S}$, Mtonga $\mathrm{C}$ : Inequities in the global health workforce: the greatest impediment to health in sub-Saharan Africa. Int J Environ Res Public Health 2007, 4:93-100.

14. Salicrup L, Fedorková L: Challenges and opportunities for enhancing biotechnology and technology transfer in developing countries. Biotechnol Adv 2006, 24:69-79.

15. Shortell SM, O'Brien JL, Carman JM, Foster RW, Hughes EF, Boerstler H, O'Connor EJ: Assessing the impact of continuous quality improvement/ total quality management: concept versus implementation. Health Serv Res 1995, 30:377-401.

16. Wass V, Van D, Shatzer J, Jones R: Assessment of clinical competence. Lancet 2001, 357:945-949.

17. Prislin M, Giglio M, Lewis E, Ahearn S, Radecki S: Assessing the acquisition of core clinical skills through the use of serial standardized patient assessments. Acad Med 2000, 75:480-483.

18. Coleman M, Nasraty S, Ostapchuk M, Wheeler S, Looney S, Rhodes S: Introducing practice-based learning and improvement ACGME core competencies into a family medicine residency curriculum. Jt Comm Qual Saf 2003, 29:238-247.

19. Kyeyagalire R: Data Management Collaborative - Uganda. [http://www. hciproject.org/node/1420].

20. Clifford GD, Blaya JA, Hall-Clifford R, Fraser HS: Medical information systems: A foundation for healthcare technologies in developing countries. Biomed Eng Online 2008, 7:18

21. Lulseged S: Integrated management of childhood illness: a review of the Ethiopian experience and prospects for child health. Ethiop Med J 2002, 40:187-201

22. Pandey $P$, Sehgal A, Riboud M, Levine D, Goyal M: Informing resource-poor populations and the delivery of entitled health and social services in rural India: a cluster randomized controlled trial. JAMA 2007, 298:1867-1875.

23. Chalmers I: Trying to do more Good than Harm in Policy and Practice: The Role of Rigorous, Transparent, Up-to-Date Evaluations. The ANNALS of the American Academy of Political and Social Science 2003, 589:22-40.

24. Björkman M, Svensson J: When is community-based monitoring effective?. Evidence from a randomized controlled experiment in Uganda; [http://didattica.unibocconi. it/mypage/upload/49950_20091016_014406_JEEA_BJORKMANSVENSSON_REVISED. PDF].

25. Haines A, Kuruvilla S, Borchert M: Bridging the implementation gap between knowledge and action for health. Bull World Health Organ 2004 82:724-731.

26. Sanders D, Haines A: Implementation Research Is Needed to Achieve International Health Goals. PLoS Med 2006, 3:e186.
27. Kotagal M, Lee P, Habiyakare C, Dusabe R, Kanama P, Epino HM, Rich ML, Farmer PE: Improving quality in resource poor settings: observational study from rural Rwanda. BMJ 2009, 339:b3488.

28. Barker PM, McCannon CJ, Mehta N, Green C, Youngleson MS, Yarrow J, Bennett B, Berwick DM: Strategies for the scale-up of antiretroviral therapy in South Africa through health system optimization. J Infect Dis 2007, 196(3):457-463.

29. Youngleson M, Nkurunziza P, Jennings K, Arendse J, Mate K, Barker P: Improving a mother to child HIV transmission programme through health system redesign: quality improvement, protocol adjustment and resource addition. PLoS One 2010, 5:e13891

30. Weiser T, Haynes A, Dziekan G, Berry W, Lipsitz S, Gawande A: Effect of a 19-item surgical safety checklist during urgent operations in a global patient population. Ann Surg 2010, 251:976-980

31. Orlander J, Barber T, Fincke B: The morbidity and mortality conference: the delicate nature of learning from error. Acad Med 2002, 77:1001-1006.

32. WHO: WHO $\mid$ Adherence to long-term therapies: evidence for action. http://www.who.int/chp/knowledge/publications/adherence_report/en/.

33. Cheng $S$, Hou Y, Chen C: Does continuity of care matter in a health care system that lacks referral arrangements? Health Policy Plan 2010, 26(2):157-162.

34. Mukherjee J, Eustache F: Community health workers as a cornerstone for integrating HIV and primary healthcare. AIDS Care 2007, :73-82.

35. Filerman G: Closing the management competence gap. Human Resources for Health 2003, 1:7.

36. Egger D, Travis P, Dovlo D, Hawken L, Policy W: Strengthening Management in Low-Income Countries.

doi:10.1186/1744-8603-8-41

Cite this article as: Maru et al:: Crossing the quality chasm in resourcelimited settings. Globalization and Health 2012 8:41.

\section{Submit your next manuscript to BioMed Central and take full advantage of:}

- Convenient online submission

- Thorough peer review

- No space constraints or color figure charges

- Immediate publication on acceptance

- Inclusion in PubMed, CAS, Scopus and Google Scholar

- Research which is freely available for redistribution

Submit your manuscript at www.biomedcentral.com/submit
C Biomed Central 